\title{
Film dynamics relevant to spray cooling
}

\author{
E. Gehring ${ }^{1}$, G. Soriano ${ }^{2}$, Y. P. Lin ${ }^{2}$, J. L. Alvarado ${ }^{2}$ \\ \& M. F. Trujillo ${ }^{1}$ \\ ${ }^{1}$ University of Wisconsin-Madison, USA \\ ${ }^{2}$ Texas A\&M University, USA
}

\begin{abstract}
For a number of years spray cooling has shown to be a viable alternative for thermal management of high-density electronics. Nevertheless, the key fundamental physical processes are to a large degree poorly understood due mostly to the complicated fluid dynamics resulting from nucleate boiling coupled with spray drop impingement. In this combined experimental and modeling effort, a representative configuration consisting of a liquid film resting on a solid silicon-based substrate with an imposed constant heat flux and an impinging train of droplets has been studied. This configuration mimics to a great degree the physics of spray cooling, while simultaneously simplifying the experimental and computational analysis to a manageable level. It is shown that a number of statistically quasi-stationary states are possible by carefully coordinating the heat flux and drop impingement rates. Studies were both performed for water and FC-72. Due in part to its lower surface tension, the quasi-stationary states for FC-72 were instantaneously much more chaotic than the corresponding water cases. The OpenFoam (open source computational fluid dynamics) code has been supplemented with an energy equation within the existing Volume-of-Fluid infrastructure. This was used to analyze the dynamics in the impingement region. It is shown that the temperature in this region is approximately equal to the temperature of incident droplets. For all water and FC-72 films, it was found that each droplet impact penetrated the entire thickness of the film bringing a significant cooling effect on the heated substrate. This was the case even for film thickness-to-impact droplet diameter ratios far exceeding one.
\end{abstract}

Keywords: spray cooling, drop impingement, heat transfer enhancement, twophase flow. 


\section{Introduction}

Current trends in today's technological developments are geared toward miniaturization of electric and electronic devices, which inherently places a significant demand on the ability to dissipate heat economically, while still maintaining an adequate temperature environment. Among the means of exercising this phase-change technology, spray cooling can achieve peak heat fluxes that can be several times higher than saturated pool boiling critical heat flux [1]. Due to this attractive means of enhancing heat transfer, spray cooling has received substantial attention as reported by numerous experimental studies and a few computational investigations. For instance, the work of Pais et al. [6], Sodtke and Stephan [9], Hsieh and Yao [3], and Selvam et al. [8] have elucidated some aspects of spray cooling. A recent review of the state of the art in spray cooling is provided by Kim [5], where the author summarizes some of the key findings in the body of work. Interestingly enough, a recurring conclusion in the literature is that the physics of spray cooling are insufficiently understood due to complexity of the interplay between multiple drop impingement and bubble nucleation, rendering the heat transfer processes in the thin liquid film extremely chaotic.

One promising avenue for uncovering the interplay between the dominant physical mechanisms in spray cooling is to remove the randomness in incident droplet size, frequency of arrival, and velocity at impingement. This can be achieved by carefully controlling the diameter and velocity of incident droplets, through the use of a piezoelectric droplet generator. Under this configuration, key non-dimensional quantities such as Weber and Reynolds number as well as the ratio of film height to droplet diameter, among others, can be manipulated at will. In the current work, a representative configuration consisting of a liquid film resting on a solid silicon-based substrate with an imposed constant heat flux and an impinging train of droplets is studied. The work presented here represents on-going efforts in both numerical simulations and experiments. In what follows a description of the experimental apparatus and procedure is presented. Details regarding the direct numerical simulations are given along with a description of the numerical framework employed. Results for water and FC-72 films are presented followed by conclusions and summary.

\section{Experimental apparatus}

\subsection{Experimental setup}

The experimental apparatus consisted of a piezo-electric droplet generation system, a heater system and an imaging system. The fluid was delivered by a syringe pump and was disturbed by high frequency vibrations from a piezoelectric electric crystal attached to an orifice plate. The high frequencies were controlled and delivered by a frequency signal generator. The set up was able to generate monodispersed stream of droplets ranging from 50 to $300 \mu \mathrm{m}$ with exit velocities from 1 to $30 \mathrm{~m} / \mathrm{s}$. 
For the heat transfer experiments, the droplet generator was able to generate water droplets in the order of 80 to $130 \mu \mathrm{m}$ using a $50 \mu \mathrm{m}$ orifice plate. In the case of FC72, droplets were in the order of 200 to $260 \mu \mathrm{m}$ using a $150 \mu \mathrm{m}$ orifice plate. The heater system consisted of a ceramic heater, copper block and a silicon wafer which received the impact of the stream of droplets. The ceramic heater was located at the base of the set up, followed by the copper block which was used to ensure uniform heat flux. The silicon-based wafer was placed on top of the copper block. The high power density ceramic heater capable of providing up to $900 \mathrm{~W}$ with a $\mathrm{K}$ type thermocouple embedded in it. The heater was joined to a copper block using a high thermal conductivity paste. The copper block had two T-type thermocouples embedded located at the geometrical center of the block at different heights to validate the heat flux value, and to estimate temperature on the wafer surface as explained below. The copper block was thermally isolated using Glastherm grade HT200 insulating material ideal for humid and wetting conditions. The power was supplied through a lambda GEN $1500 \mathrm{~W}$ and the temperatures were logged using an OMEGA-DAQPRO-5300 data acquisition system. The thermal resistance from the heater to the top of the silicon wafer was determined by measuring the temperature difference between a thin film thermocouple (removed during the cooling experiments) attached to the wafer, and a thermocouple attached to the heater. During the experiments, the thermal resistance was used to estimate the wafer's surface temperature using the thermocouples embedded in the copper block.

The imaging system consisted of a high speed Photron SA3 camera capable of recording images at 1,000 frames per second (fps) at a resolution of 1,024 $\mathrm{x}$ 1,024 pixels, and up to $60,000 \mathrm{fps}$ at a reduced resolution of $128 \times 16$ pixels. The camera consisted also of a zoom 6000 series lens from Navitar capable to work with coaxial and backlight illumination, and attachments that were used to provide the required magnification to observe individual droplets at a magnification of $18 \mathrm{X}$, and a magnification of $0.35 \mathrm{X}$ to capture the total dimension of the film.

\subsection{Experimental procedure}

The diameter of the droplets was measured using a particle image analysis tool from National Instruments. The image analysis tool was used to measure the projected surface area of each droplet which in turn was used to compute the droplet diameter, Sauter mean diameter and the corresponding standard deviation. The impact velocities below $3 \mathrm{~m} / \mathrm{s}$ were directly measured by tracking the position of the centroid of the droplet $1 \mathrm{~ms}$ before the position of impact. In the case of impact velocities above $3 \mathrm{~m} / \mathrm{s}$ an indirect measurement of the velocity was taken by measuring the diameter of the outgoing liquid jet, and the distance between the droplet generator head to the surface of the silicon wafer, and the total flowrate. Drag effects were estimated and found to be negligible.

The experiment consisted in heating the surface initially until it reached a desired temperature using a constant power input, then droplets were allowed to impinge the surface of the heater. The heat flux and fluid flow rate were adjusted 
simultaneously until a steady liquid film was formed. The distance of the droplet generator head to the heaters was fixed at $2.4 \mathrm{~cm}$. Temperatures were recorded each second, and it was considered that the system reached steady state when the temperature changes remain in a range of $\pm 0.5^{\circ} \mathrm{C}$ for at least $3 \mathrm{~min}$. During the steady state phase, images of the film were captured using the imaging system at an angle of $90^{\circ}$ and $20^{\circ}$ with respect to the surface of the heater.

The images taken were analyzed using the image processing toolbox of Matlab ${ }^{\circledR}$. The images at an angle of $90^{\circ}$ were used to obtain the rms contact angle of the film, and the rms values of the film height at 4 fixed positions of the film. The images obtained at angle of $90^{\circ}$ and $20^{\circ}$ were used to obtain the average diameter, and area of the film.

\section{Numerical simulation}

Due to the chaotic nature of droplet impingement resulting in splashing and film fragmentation, an implicit surface tracking capability was employed using the Volume-of-Fluid (VoF) methodology (Hirt and Nichols [2]) with added interfacial compression for sharp interface capturing. The basis for our calculations was a modified version of the flow solver, interFoam, which belongs to a class of open source computational fluid dynamics solvers, OpenFOAM (Open Field Operation and Manipulation) [4]. Our modifications primarily consisted of the addition of an energy equation within the VoF treatment as described below.

The governing equations are continuity and momentum, namely

$$
\begin{gathered}
\nabla \cdot \mathbf{u}=0, \\
\frac{\partial \rho \mathbf{u}}{\partial t}+\nabla \cdot(\rho \mathbf{u} \otimes \mathbf{u})=-\nabla p^{*}+\nabla \cdot(\mu \nabla \mathbf{u})+\nabla \mathbf{u} \cdot \nabla \mu-\mathbf{g} \cdot \mathbf{x} \nabla \rho+\sigma \kappa \nabla \gamma,
\end{gathered}
$$

where $\mathbf{u}$ is the velocity vector, $t$ is time, $\mathbf{x}$ is the coordinate vector, $p^{*}=p-\rho \mathbf{g} \cdot \mathbf{x}$ is the modified pressure, $\mathbf{g}$ is the gravity vector, $\sigma$ is the surface tension, $\kappa$ is the curvature of the interface, and $\gamma$ is the volume fraction, which is used to compute the mixture density and viscosity

$$
\begin{aligned}
& \rho=\gamma \rho_{l}+(1-\gamma) \rho_{g}, \\
& \mu=\gamma \mu_{l}+(1-\gamma) \mu_{g} .
\end{aligned}
$$

Additionally, the free surface was computed by solving a volume fraction transport equation $(\mathrm{VoF})$

$$
\frac{\partial \gamma}{\partial t}+\mathbf{u} \cdot \nabla \gamma=-\nabla \cdot\left(\mathbf{U}_{r} \gamma[1-\gamma]\right),
$$

where, an additional right-hand-side term was used to compress the interface and thus minimize numerical diffusion. In (6), $\mathbf{U}_{\mathbf{r}}$ is a velocity field normal to the interface, which was used to enforce this compression. It is defined by 


$$
\mathbf{U}_{r}=k_{c} \mathbf{n} \max \left(\frac{|\mathbf{n} \cdot \mathbf{u}|}{|S|^{2}}\right)
$$

Here $k_{c}$ is an adjustable coefficient used to adjust the amount of compression (set normally to 1). The interface unit normal $\mathbf{n}$ is computed by taking the gradient of a smoothed (via elliptic relaxation) volume fraction $\gamma_{f}$ at the cell faces.

$$
\mathbf{n}=\frac{\nabla \gamma_{f}}{\left|\nabla \gamma_{f}\right|} .
$$

Due to the term $\gamma(1-\gamma)$ in (5), the interfacial compression was only active near the interface. Numerical tests reveal that the solution of (5) was mass conservative.

The energy equation, which has been added to the aforementioned interFoam solver, represents the conservation of energy for an incompressible two-phase mixture within the context of the VoF scheme. It is given as

$$
\frac{\partial(C \rho)^{I} T}{\partial}+\nabla \cdot\left[(C \rho)^{h} \mathbf{U} T\right]=k^{m i x} \nabla^{2} T
$$

For the moment we have not considered phase change; the necessary code modifications to handle this generalization are currently underway. In $(9),(C \rho)^{\mathrm{I}}$ and $(\mathrm{C} \rho)^{\mathrm{h}}$ are phase weighted quantities and are defined as follows,

$$
\begin{aligned}
& (C \rho)^{I}=C^{l} \rho^{l} \gamma+C_{v}^{g} \rho^{g}(1-\gamma) \\
& (C \rho)^{h}=C^{l} \rho^{l} \gamma+C_{p}^{g} \rho^{g}(1-\gamma) .
\end{aligned}
$$

The conductivity is calculated in a similar way, using the liquid volume fraction.

$$
k^{m i x}=k^{l} \gamma+k^{g}(1-\gamma) .
$$

In summary, equations (1) through (5) were used to simulate the fluid flow in the calculations presented in this paper. The solution began with the calculation of the phase interface by which the liquid volume fraction was obtained. The liquid volume fraction, equation (5), was discretized using a finite volume method and was solved using a MULES (Multidimensional Universal Limiter with Explicit Solution) scheme. This scheme is specially designed for solving convective transport equations. In this context, MULES was used as an explicit solver using a first order Euler scheme for marching in time and the CFL number was restricted to 0.5 . The material properties, density, conductivity, viscosity, and the product of specific heat and density were updated according to the position of the interface at each time step using the liquid volume fraction as described by (3), (4), and (10). Then, momentum was solved, (2), and the pressure field was subsequently calculated using an implicit pressure-velocity 
coupling algorithm. A PBiCG (Preconditioned Bi-Conjugate Gradient) solver with a DILU (Diagonal Incomplete-LU) preconditioner was used to solve for the momentum equation. A PCG (Preconditioned Conjugate Gradient) with a DIC (Diagonal Incomplete-Cholesky) preconditioner was used to solve for the pressure field. Finally, the energy equation (8) was solved using a BICCG (Diagonal incomplete LU preconditioned BiCG) solver. The advection term in the energy equation was solved using the SuperBee scheme.

\section{Results}

Experimental and computational results for water and $\mathrm{FC}-72$ are presented in the following subsections of the paper. In general, the process can be described as exhibiting two distinct spatial regions. Macroscopically, the liquid film underwent vigorous pool boiling with large bubbles disrupting and in some cases atomizing the free surface. Even though the process was highly unsteady, from a statistical point of view, the average values for the dimensions of the film were to a large degree stationary. At a more local level, the impact region for the train of the droplets occurred near the centre of the film. A much thinner liquid film void characterized it. It is hypothesized that due to the large fluid velocities emanating from drop impact in this region, the growth of bubbles was severely disrupted.

\subsection{Water cases}

Experiments were performed using deionised water, which was injected at room temperature. Flow rates of 70 and $110 \mathrm{ml} / \mathrm{hr}$ were used. For each flow rate, a constant heat input was kept, and changes in surface temperatures and film parameters were recorded. Table 1 summarizes the experimental data. Cases 1 and 2 corresponded to $70 \mathrm{ml} / \mathrm{hr}$, where changes in droplet velocity and size were

Table 1: $\quad$ Heat transfer results of water.

\begin{tabular}{|c|c|c|c|c|}
\hline Parameters & Case 1 & Case 2 & Case 3 & Case 4 \\
\hline Flow rate $(\mathrm{ml} / \mathrm{hr})$ & 70 & 70 & 110 & 110 \\
\hline Frequency of Droplets $(\mathrm{Hz})$ & 19725 & 44219 & 34901 & 105330 \\
\hline Velocity $(\mathrm{m} / \mathrm{s})$ & 9.6 & 9.7 & 15.3 & 15.3 \\
\hline Droplet diameter $(\mu \mathrm{m})$ & 123.5 & 94.4 & 118.7 & 82.1 \\
\hline Diameter standard deviation $(\mu \mathrm{m})$ & 3.0 & 2.1 & 4.3 & 2.7 \\
\hline Weber number & 160 & 123 & 385 & 282 \\
\hline $\mathrm{T}_{\mathrm{s}}(\mathrm{K})$ & 396 & 400 & 395 & 396 \\
\hline Heat dissipated $(\mathrm{W})$ & 239 & 240 & 332 & 331 \\
\hline Heat flux $\left(\mathrm{W} / \mathrm{cm}^{2}\right) *$ & 11.8 & 11.9 & 16.4 & 16.4 \\
\hline Diameter impact zone $(\mu \mathrm{m})$ & 751 & 486 & 1141 & 743 \\
\hline Film Thickness impact zone $(\mu \mathrm{m})$ & $17-25$ & $18-26$ & $32-60$ & $35-45$ \\
\hline Ratio impact zone diam./drop diam. & 6.1 & 5.2 & 9.6 & 9.0 \\
\hline
\end{tabular}

*Based on a 1D thermal conductivity model and it is assumed to be the average for an entire heater. 
performed with the objective of varying the Weber number. A larger impact zone was recorded for the higher Weber number case. The resulting surface temperature values showed little variation with respect to changes in this nondimensional quantity. Cases 3 and 4 corresponded to another stationary state for the liquid film. The impact zone was similarly larger for the higher Weber number case and much larger in comparison to cases 1 and 2. This was likely due to higher flow rates used. Nevertheless, the recorded temperature values were not only similar in magnitude but were surprisingly similar to cases 1 and 2. It should be noted that these temperature values were taken some distance below the solid substrate and were based on an average thermal resistance; hence, they represented a global characterization of the film temperature.

A likely conclusion regarding the temperature behaviour is that for conditions where a stationary state has been reached between the small cooling effect of a single train of impinged droplets and the heat flux applied, the recorded temperature only represents a reference point. A higher heat flux could have lead to a higher temperature but only at the expense of a departure from the stationary state into a dry out condition. In the same manner, a decrease in the heat flux would have potentially driven the system into a lower temperature condition, subsequently promoting flooding and complete pool boiling conditions and a loss of the stationary state. Hence, a value for the global temperature of approximately $393 \mathrm{~K}$ was characteristic of this stationary state. Currently an infrared temperature sensing equipment is being developed to be able to measure surface temperature spatially.

In spite of the usefulness of performing the aforementioned film measurements in studying the thermal and kinematic behavior of droplet train impingement, the more immediate physics in the impact zone were still to a large degree illusive. These measurements can only give us global information on the temperature conditions from which only indirect information regarding the local impingement behavior can be deduced. To address this issue, an application of the Laser Induced Fluorescence (LIF) is currently being put in place. Experimental results from this technique are forthcoming. Computationally, however, this region was targeted for direct numerical simulation in the present work and the results presented in what follows.

The overall computations were performed using parallel computing on 64 processors with a grid size of approximately $12 \mu \mathrm{m}$ with nearly isotropic cell quality. The mesh consisted of approximately $6 \times 10^{6}$ computational cells. The computational domain was cylindrical with a diameter equal to 20 droplet diameters; its height was $6 \mathrm{~mm}$. This resolution was sufficiently large such that boundary effects were assumed to not affect the dynamics in the impact zone. All boundary surfaces with the exception of the top surface, which was open to the atmosphere, were assigned no-slip wall boundary conditions. All of the properties, including density, viscosity, surface tension, droplet velocity and size, film thickness, conductivity, specific heat, and applied heat flux matched the experimental properties and conditions discussed previously. The domain was initialized with a train of droplets and the case was run some time after all the droplets had impacted the pool of liquid. The number of droplets ranged from 
11 droplets in case 1 to 37 droplets in case 4 . Note that these cases corresponded to the cases with the lowest and highest droplet frequencies.

Four different cases corresponding to the conditions reported in Table 1 (i.e. heat flux, droplet flow rate, diameter, and velocity) were computed. The heat flux was applied at the bottom of the domain and a pool of liquid $200 \mu \mathrm{m}$ thick was used to initialize the computation. The initial droplet temperature was $290^{\circ}$ $\mathrm{K}$ for all water cases. A qualitative comparison between the experiments and computations is shown in Figure 1 for one flow rate. The overall features of the crown were well simulated in the computations to the extent that ring structures in the lower portion of the impact point were captured. At higher flow rates the radial dimension of the impact zone, characterized by a thin liquid film, increased substantially. This behaviour was also observed in the computations.

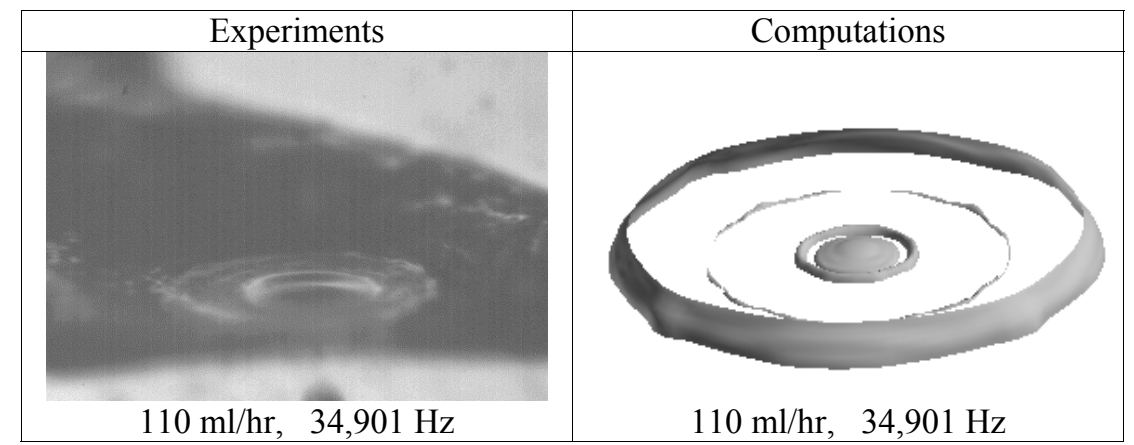

Figure 1: Qualitative comparison between experimental and computational images of a train of water droplets impacting a pre-wetted, heated surface. The flowrate was $110 \mathrm{ml} / \mathrm{hr}$ with a droplet injection frequency of 34,901 .

The temperature at the point of droplet impact (geometrical center of domain) was recorded. Beyond an initial transient period, very small magnitude oscillations with a frequency consistent with the droplet frequency of impingement were observed. This is shown more clearly in figures $2 a$ and $2 b$, corresponding to cases 1 and 4, respectively. Even though the droplet velocity and diameter were of similar magnitude, between cases 1 and 4, the histories showed consistent rather larger temperature oscillations for the lower frequency impingement conditions. This was caused by a larger time period between successive drop impacts, which allowed more time for surface temperature recovery. This resulted in lower mean temperature values for the higher frequency cases. The range in mean temperature differences for all cases were within $0.05^{\circ} \mathrm{K}$, which was virtually insignificant in comparison to the difference between the initial droplet temperature and the overall film temperature. This demonstrated that at the center of droplet impact, convection was sufficiently strong to reduce the local temperature to the initial droplet temperature value. Moreover, this behavior was independent of Weber number and frequency of 


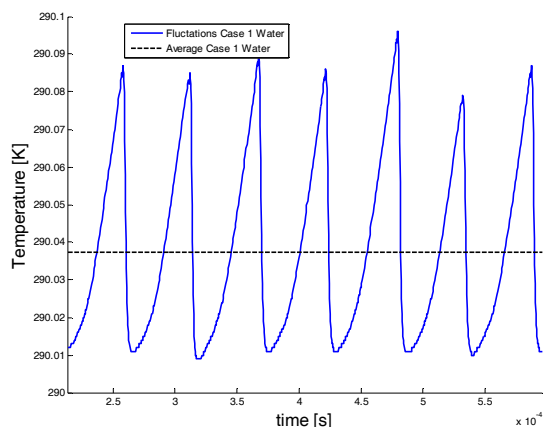

(a)

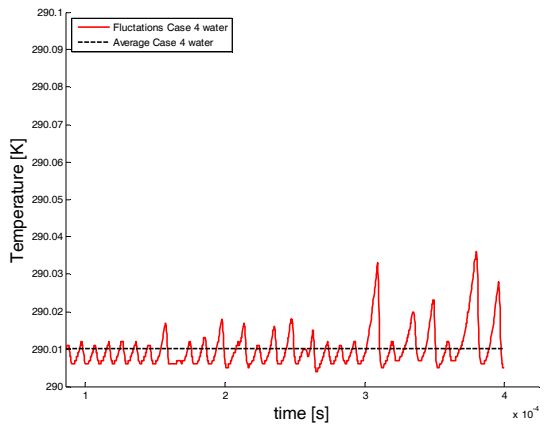

(b)

Figure 2: Comparison of temperature histories at the center of the impact zone for water case 1 a) (flow rate $=70 \mathrm{ml} / \mathrm{hr}$, frequency $=19,725$ $\mathrm{Hz}$, Weber $=160)$ and case $4 \mathrm{~b})($ flow rate $=110 \mathrm{ml} / \mathrm{hr}$, frequency $=$ $105,330 \mathrm{~Hz}$, Weber $=282$ ).

impact for the range of conditions studied here. For much lower frequencies, it can be expected that deviations from these observations would likely be obtained. In the vicinity of droplet impact, the same conclusions hold, with the exception that the mean values were $\mathrm{O}(10) \mathrm{K}$ higher due to heating that occurred as the liquid traveled from the center of droplet impact to the periphery of the domain. In comparison to the experimentally obtained values (Table 1), which represented global behavior, the local temperature in the vicinity of the impact point was significantly lower. This is expected due to the much higher convection heat transfer.

\subsection{FC-72 cases}

The experimental procedure for FC-72 was essentially the same as for water, with the exception that different flow rates, heat fluxes, and temperatures were recorded for the stationary states, due to differences in physical properties. The data are summarized in Table 2. For cases 1 and 2, and for cases 3 and 4, a flow rate of 120 and $150 \mathrm{ml} / \mathrm{hr}$ was used, respectively. The temperature variations were minimal around a value of $359{ }^{\circ} \mathrm{K}$. Hence, a similar mechanism of stationary states, as found for water, was also found for FC-72.

Dynamically, however, the morphology of the FC-72 film exhibited a significant increase in the level of fluctuations in comparison to water. The ratio of film height to Sauter mean diameter was on the order of 20:1 for water; while it was 5:1 for FC-72. This lower FC- 72 film thickness seems to have caused significant film instabilities during droplet impact. The differences in surface tension (water $0.072 \mathrm{~N} / \mathrm{m}$ at STP and FC-72 $0.010 \mathrm{~N} / \mathrm{m}$ ) were likely the main reasons for the observed behaviour. The role of bubble nucleation in promoting the film stabilities remained unidentified and will be pursued in future tests. Due to the more pronounced irregularity of the film shape, a number of images in the 
322 Advanced Computational Methods and Experiments in Heat Transfer XI

Table 2: $\quad$ Heat transfer and film measurement of FC72.

\begin{tabular}{|c|c|c|c|c|}
\hline Parameters & Case 1 & Case 2 & Case 3 & Case 4 \\
\hline Flow rate $(\mathrm{ml} / \mathrm{hr})$ & 120 & 120 & 150 & 150 \\
\hline Frequency of Droplets $(\mathrm{Hz})$ & 4186 & 3912 & 5053 & 8672 \\
\hline Velocity $(\mathrm{m} / \mathrm{s})$ & 2.1 & 2.1 & 2.6 & 2.6 \\
\hline Droplet diameter $(\mu \mathrm{m})$ & 247.8 & 253.4 & 250.7 & 209.4 \\
\hline Diameter standard deviation $(\mu \mathrm{m})$ & 9.4 & 1.5 & 8.3 & 5.6 \\
\hline Weber number & 189 & 192 & 288 & 240 \\
\hline $\mathrm{T}_{\mathrm{s}}(\mathrm{K})^{*}$ & 358.4 & 359.2 & 359.7 & 364.4 \\
\hline Heat dissipated $(\mathrm{W})$ & 55.0 & 54.7 & 67.2 & 64.9 \\
\hline Heat flux $\left(\mathrm{W} / \mathrm{cm}^{2}\right) *$ & 2.7 & 2.7 & 3.3 & 3.2 \\
\hline Diameter impact zone $(\mu \mathrm{m})$ & 994 & 1084 & 1299 & 1181 \\
\hline Film Thickness impact zone $(\mu \mathrm{m})$ & $30-68$ & $36-70$ & $21-75$ & $25-70$ \\
\hline Ratio impact zone diam./drop diam. & 4.0 & 4.3 & 5.2 & 5.6 \\
\hline
\end{tabular}

*Based on a 1D thermal conductivity model and it is assumed to be the average for an entire heater.

impact region were selected and used for imaging analysis. These were reduced in order to calculate the film parameters listed in Table 2.

These cases were simulated using parallel computing on 48 processors. Since the incident droplets were almost twice as large as the case for water, the total number of computational cells employed was reduced to $1.4 \times 10^{6}$. Again, we only focussed on the impact zone, neglecting the surrounding pool boiling region. All properties, including density, viscosity, surface tension, droplet velocity and size, film thickness, conductivity, specific heat, and applied heat flux matched the experimental values and conditions mentioned in Table 2. Boundary conditions corresponded to no-slip wall, with the exception of the top surface, which is open to the atmosphere. A heat flux was applied at the bottom surface and a number of droplets were numerically injected into the domain. The number of droplets ranged from 10 in case 2 to 18 droplets in case 4 . Note that these cases corresponded to the lowest and highest droplet frequencies.

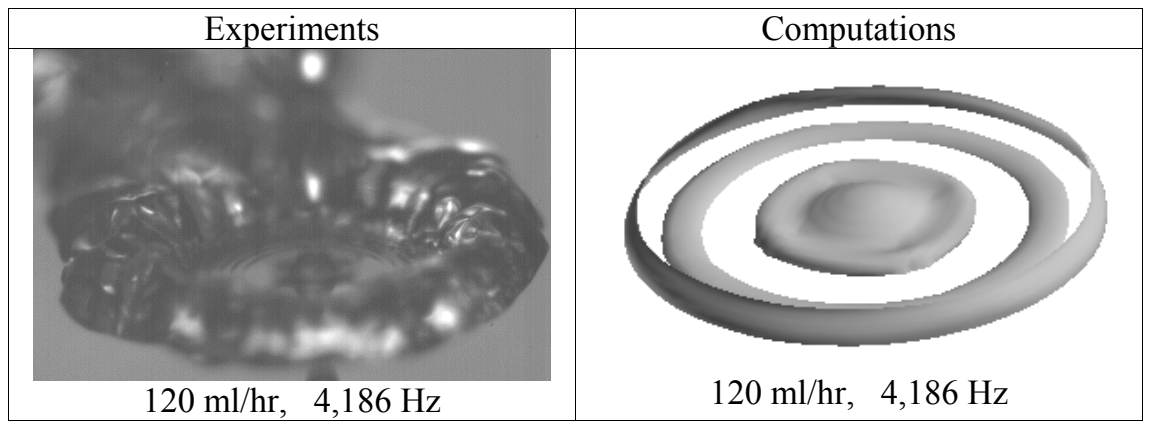

Figure 3: Qualitative comparison between experimental and computational images of a train of FC-72 droplets impacting a pre-wetted, heated surface. The flow rate was $120 \mathrm{ml} / \mathrm{hr}$ with a droplet injection frequency of $4,186 \mathrm{~Hz}$. 
Overall, a similar behaviour was observed as for the water droplet calculations. The temperature at the impact site was immediately reduced to the incident droplet temperature at impact. After this brief transient period, a number of small oscillations about this initial temperature value set in. This is shown in Figure 4. Again, for higher frequencies, the amount of time allowed for temperature recovery was minimized consequently reducing the magnitude of the fluctuations. It is interesting to observe that for both FC-72 and water, the depth of the film was immaterial, since the droplets were able to penetrate through the film, impacting the bottom surface. This provided a strong, but local, cooling effect. Nevertheless, a more pronounced crown at the point of impact can be observed in the case of FC-72 than in water which can be attributed to larger droplet size.

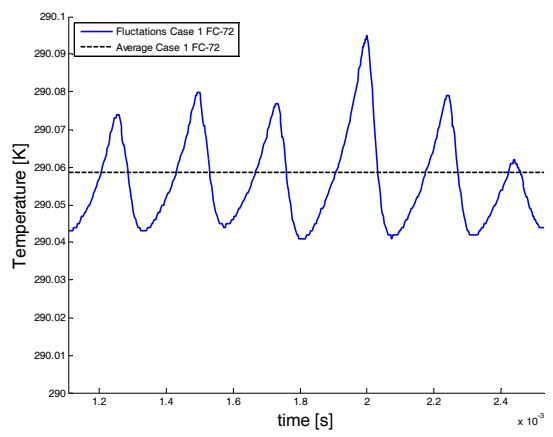

(a)

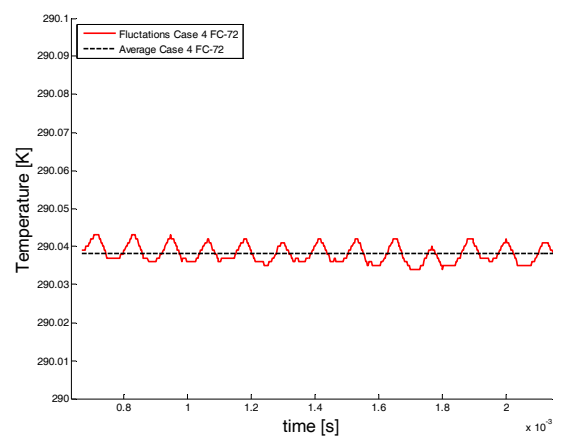

(b)

Figure 4: Comparison of temperature histories at the centre of the impact zone for FC-72 case 1 a) (flow rate $=120 \mathrm{ml} / \mathrm{hr}$, frequency $=4,186$ $\mathrm{Hz}$, Weber $=189)$ and case $4 \mathrm{~b})($ flow rate $=150 \mathrm{ml} / \mathrm{hr}$, frequency $=$ $8672 \mathrm{~Hz}$, Weber $=240$ ).

\section{Conclusions}

A preliminary investigation of the physics of spray cooling was presented here for a configuration consisting of a liquid film and an impinging train of droplets. A number of quasi-stationary states were obtained experimentally, characterized by a careful balance between the heat flux and droplet flow rate. The measured temperature was almost invariant to either changes in heat flux, flow rate, or Weber number. It is postulated that the reason for this behaviour in apparently different operating conditions reflects the nature of quasi-stationary states as limiting points in the heat transfer dynamics. For instance, increases or decreases in heat flux would likely lead to significant temperature variations but would automatically result in the loss of the stationary state. Computationally, the centre region of the film was directly simulated. The OpenFoam code was modified with the inclusion of an energy equation and was coupled to the VoF code structure. The droplet impingements were able to penetrate throughout the entire film, bringing cooler liquid directly to the underlying surface. This was the 
case even when the ratio of initial film height to incident droplet diameter largely exceeded one. The result was a temperature level at the impact zone that displayed a value roughly equal to the incident droplet temperature. Qualitatively, an agreement between experiments and computations is noted in this impingement region. Subsequent work in this topic will be aimed at analyzing the heat transfer characteristics near the wall during impingement using laser induced fluorescence and an infrared temperature sensing technique.

\section{Acknowledgements}

The authors are indebted to the support of the Thermal Management Program (Code 331) of the United States Office of Naval Research managed by Dr. Mark Spector.

\section{References}

[1] Bar-Cohen, A., Arik, M., and Ohadi, M. Direct Liquid Cooling of High Flux Micro and Nano Electronics Components, Proceedings of the IEEE, 94:8 2006.

[2] Hirt, C.W. and Nicols, B.D., Volume of Fluid (VOF) Method for the Dynamics of Free Boundaries. Journal of Computational Physics, 39, pp. 201-225, 1981.

[3] Hsieh, C.-C. and Yao, S-C., Evaporative Heat Transfer Characteristics of a Water Spray on Micro-Structured Silicon Surfaces. International Journal of Heat and Mass Transfer, 49, pp.962-974, 2006.

[4] Jasak, H., Jemcov, A., and Tukovic, Z. OpenFOAM: A C++ Library for Complex Physics Simulations. International Workshop on Coupled Methods in Numerical Dynamics IUC, Dubrovnik, Croatia, Sept. 2007.

[5] Kim, J. Spray Cooling Heat Transfer: The State of the Art. International Journal of Heat and Fluid Flow 28, pp.753-767, 2007.

[6] Pais, M., Tiltion, D. and Chow, L., High heat flow, low superheat evaporative spray cooling. AIAA-89-0241, 27 ${ }^{\text {th }}$ Aerospace Sciences Meeting, American Inst. of Aeronautics and Astronautics, Reno, Nevada, 1989, pp. 1-10.

[7] Sawyer, M.L., Jeter, S.M. and Abdel-Khalik, S.I., A critical heat flux correlation for droplet impact cooling. International Journal of Heat and Mass Transfer, 40, pp. 2123-2131, 1997.

[8] Selvam, R.P., Lin, L. and Ponnappan, R. Computational Modeling of Spray Cooling: Current Status and Future Challenges. Proceedings of Space Technology and Applications International Forum, AIP Conference Proceedings, 746, Melville, NY, 2005, pp. 56-63.

[9] Sodtke, C. and Stephan, P. Spray Cooling on Micro Structured Surfaces. International Journal of Heat and Mass Transfer, 50, pp.4089-4097, 2007. 